\title{
Power Flow Control of Renewable Energy Based Distributed Generators Using Advanced Power Converter Technologies
}

\author{
Chao-Tsung Ma and Tzung-Han Shr
}

\begin{abstract}
This paper describes a design concept in which a digital controlled power interface embedded with a battery energy storage system (BESS) named battery interfaced static synchronous generator (BISSG) is constructed to improve the power quality of micro-grid (MG) systems. Some distributed generation (DG) systems, e.g., the wind turbine generator (WTG) and the Photovoltaic (PV) systems conventionally generate real power based on natural conditions thus their output power are fluctuating from time to time. To eliminate this shortcoming, the proposed BISSG attempts to smooth the output power of DG with fast charging and discharging its BESS. To achieve a cost-effective design, the proposed BISSG is designed to maximize its control capability in terms of bilateral real power regulation and reactive power compensation for MG voltage support or power factor correction. It is important to note that the proposed BISSG is able to fulfill various real power dispatching functionalities required by the system operator. In this paper, the mathematical model of BISSG and its related controllers are firstly addressed. Then, simulation studies and hardware tests on a simplified MG network are carried out. Typical results are presented with brief discussions to demonstrate the feasibility and effectiveness of the proposed control scheme.
\end{abstract}

Index Terms - Renewable energy, wind turbine generator, battery interfaced static synchronous generator, distributed generation, micro-grid.

\section{INTRODUCTION}

With the rapid development of new energy technologies, more and more renewable energy sources are being integrated into power distribution systems in the form of distributed generations (DG). In recent years, power converter interfaced DG systems, renewable energy sources (RES) based micro-grids (MG) and state-of-the-art communication and control technologies have been recognized to play important roles in the development of future energy policies. Short-term goals of these energy policies include reduction in high-polluting power generations and global greenhouse gas emissions, improved diversity and security of energy supply, and exploitation of possible incentives for creating local value added opportunities for the related industrial sectors. Based on the related technical reports in the open literature, potential

Manuscript received September 25, 2013; revised January 16, 2014. This work was supported in part by the National Science Council of Taiwan, R.O.C. under Grant NSC102-2221-E-239-018.

Chao-Tsung Ma and Tzung-Han Shr are with the Electrical Engineering Department, National United University, Miao-Li City 36003, Taiwan, R.O.C. (tel.: 886-37-381369; fax: 886-37-327887; e-mail: ctma@nuu.edu.tw).
RES based power generations may include wind turbine generator, photovoltaic and fuel cell systems [1]-[3]. Of these power generating methods, the interests in photovoltaic (PV) and wind turbine generator (WTG) are growing worldwide. The annual growth rate is counted at $25-35 \%$ for $\mathrm{PV}$ and about $20-23 \%$ for WTG over last five years. It is recorded roughly $25 \mathrm{GW}$ of total commercial PV installations and about $270 \mathrm{GW}$ for WTG at the beginning of 2013 and the increasing trend of using PV and WTG systems still continues [4]. In fact, a number of different PV and WTG incentive programs have been introduced in Taiwan since 2008 and most of the developed countries are currently promoting residential and commercial uses of PV and WTG generation systems [5]-[7]. Based on the standards such as IEEE1547, IEEE 929 and UL1741, DG inverter systems should operate at unity power factor [8]; however, this regulation has some limitations in practical applications, especially when some system-level control functions are to be performed. On the other hand, considering the installation quantity of PV and WTG has been continuously increased in recent years and the WTG and the PV systems generate real power based on nature conditions thus their output powers are not stable; this leads to a problem that fast output power fluctuation of the DG with high capacity can cause network frequency and voltage variations, especially in isolated power systems, e.g., a MG operated in autonomous mode and thus impairing the power quality of the local power systems [9]. This problem becomes even more crucial in WTG as wind is normally intermittent and stochastic in fast dynamic feature.

In the open literature, there have been a number of methods proposed to smooth the wind farm output power flow. Most of the reported methods are using an energy storage system (BESS) to absorb or provide real power on various control algorithms. Potential technical issues, concerning different aspects related with the management of WTG systems and their integration into the distributed network, have been discussed in [10]-[12]. In those reported methods, the major difficulty in WTG output power smoothing control is the setting of its output power command. Using a constant output power reference based on historical WTG power generation data is easy but not reliable because there can be some cases where wind speed is very low and then the logged power command cannot be achieved. Theoretically, the BESS is able to solve the problem but large energy capacity will be needed. To give flexibility in designing the control scheme, this paper proposes an easy control scheme to generate the reference output power for the MG feeder connected with WTG and thus the energy capacity of BESS unit in the BISSG can be 
economically chosen as desired. Moreover, the reactive power output of the BISSG can also be controlled to keep the WTG terminal voltage constant if desired. The above design concept with some simulation results have been reported in [13]. It is important to note that to further verify the feasibility of the design concepts, more comprehensive simulation studies have been carried out. Typical results are presented in this paper and the proposed BISSG system designed with fully digital control scheme for the integration of a variety of control functions into a single hardware architecture has been constructed in the laboratory of applied power electronics research group (APERG), National United University, Taiwan. The corresponding measured results are simultaneously presented with the simulation cases for comparative purpose.

\section{MiCRO-GRID AND ITS CONCEPTS}

Distribution systems possessing DGs and controllable loads with the ability to operate in both grid-connected and standalone modes are an important class of the so-called MG power systems [14], [15]. A typical MG system is shown in Fig. 1. As can be seen in Fig.1, the micro-grid has three independent Zones connected with the distribution system with a static switch (SS). In Zone1, there is a micro-turbine (uTG), a controllable passive load bank. In Zone2, a PV system is installed. A controllable load bank as that installed in Zone1 is also connected. Zone3 is equipped with a $4 \mathrm{~kW}$ small PMSG based wind turbine generator (WTG). To facilitate possible tests regarding the proposed power smoothing control schemes, a BISSG is installed in parallel with the PMSG based WTG for various conditions in practical MG operating modes.

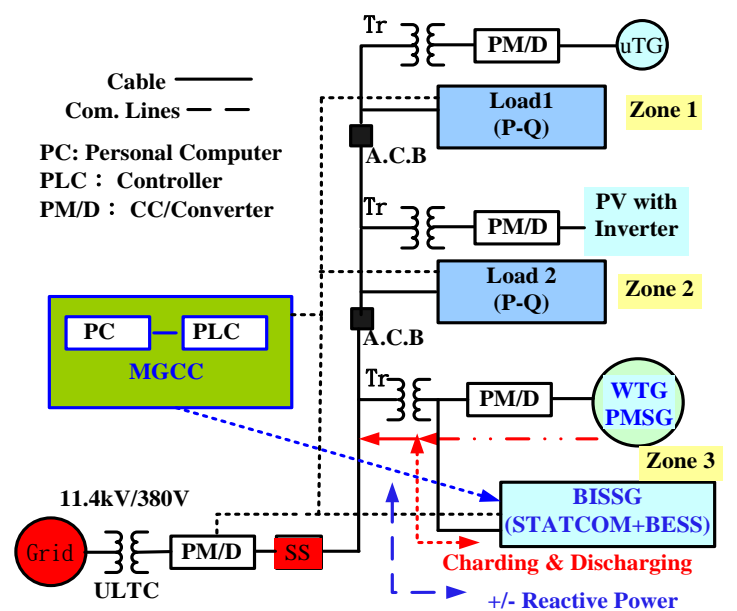

Fig. 1. System diagram of a typical micro-grid with various DG inverters and the proposed BISSG.

It is well known that MG strives for optimized operation of the aggregated distribution systems by coordinating the various DGs, BESS and load resources not only when connected to the distribution system but also in a standalone mode. In either modes of operation, advanced local controls, energy management, power quality and protection technologies are required for robustness and reliability. In practice, the energy management optimization objective function can be tailored to the needs of each application. In a practical MG project, the overall objective is to optimize operating performance and cost in the normally grid-connected mode, while ensuring that the system is capable of meeting the power quality performance requirements in standalone mode. To satisfy the needs of possible applications, some BESS units are inevitably required to achieve some advanced operations and power management functions.

\section{BISSG PRINCIPLES AND CONTROL SYSTEMS}

\section{A. BISSG Hardware Configuration}

The operating principles and control concepts of the proposed BISSG are actually derived from the static synchronous compensator (STATCOM), a popular shunt-type FACTS device or the shunt-branch of a unified power flow controller (UPFC). In this paper, a basic BISSG hardware configuration which consists of a 3-phase switching converter using 6 IGBT switches and a BESS as shown in Fig. 2 is chosen to introduce the proposed BISSG operating principles and its control schemes. The IGBT converter in the BISSG is designed to be operated from a DC link voltage provided by a battery bank. In normal operations, the active power can be controlled in either direction between the AC terminals of the converter and the grid to regulate the DC voltage of the battery bank and thus the charging and discharging function can be performed. In this hardware topology, the converter can also generate or absorb reactive power independently at its AC output terminals to affect system voltages or simply to act as a reactive power generator.

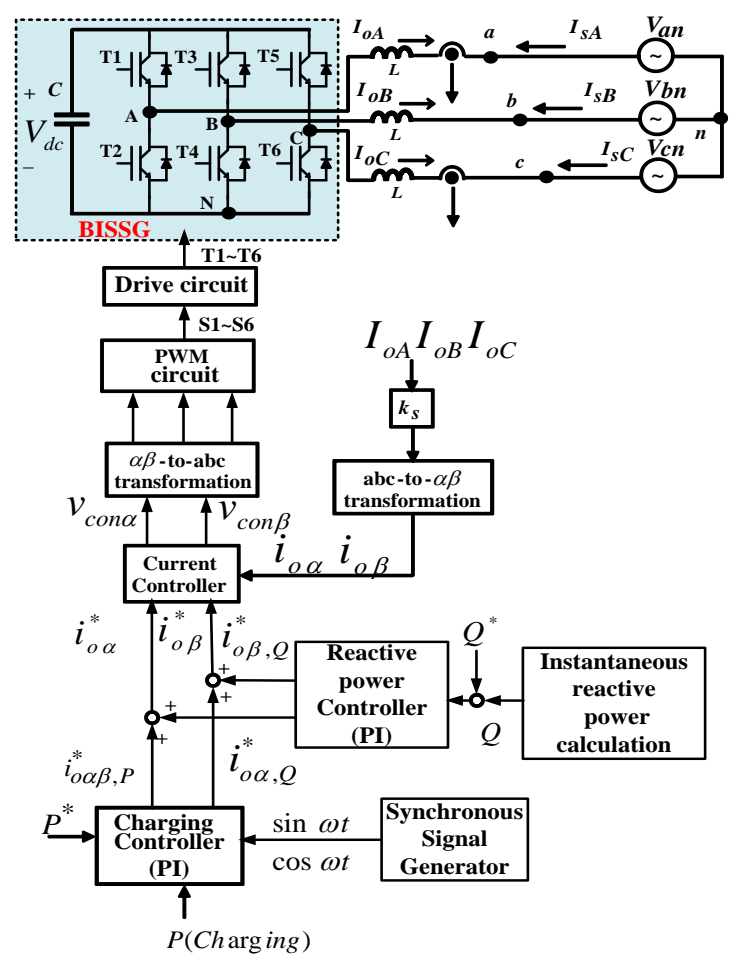

Fig. 2. A simplified MG model with the proposed BISSG and control signals

\section{B. WTG Systems and Hardware Configuration}

A typical PMSG based WTG control system is shown in Fig. 3. As can be seen in Fig. 3, the Inverter1 is in charge of MPPT control according to the wind speed; while the 
Inverter2 is used to bilaterally regulate the WTG output power via controlling the DC voltage $(V d)$. In this paper, the conventional pitch controller is not used for simplicity reason. In practice, the main purpose of using pitch controller is to maintain the output power of WTG at the rated level by controlling the blade pitch angle of the turbine when wind speed is over the rated speed.

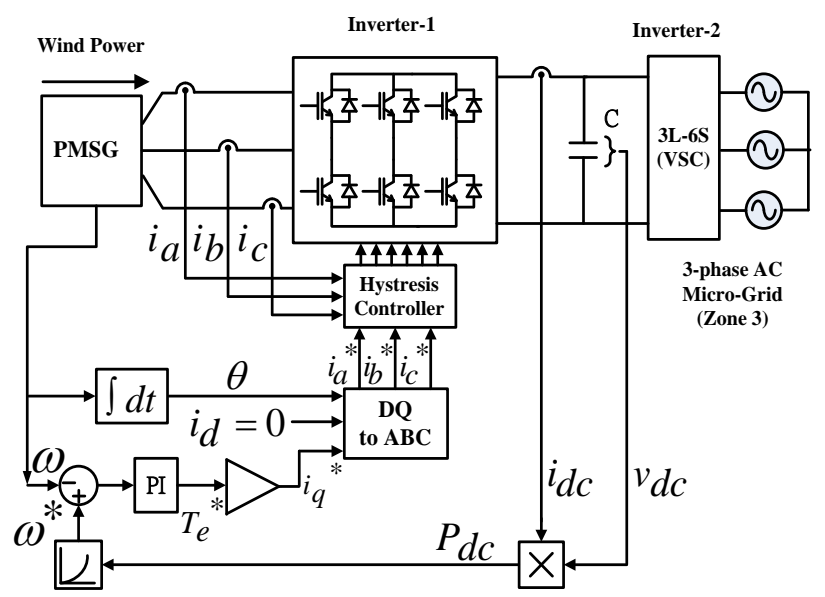

Fig. 3. A typical WTG (PMSG) hardware system with controllers.

\section{DESIGN OF BESS BASED BISSG CONTROL SYSTEMS}

In this study, two important control functions are designed for the BISSG; i.e., real power regulation for DG and reactive power compensation for the grid. These control functions can be activated simultaneously or individually. Because the proposed compensating requirements, i.e., real and reactive power regulations, are directly related to the currents, shunt-type connection of DG inverter is a realistic topology as it normally injects currents at PCC.

Therefore, this study uses the shunt-type connecting format for the implementation of BISSG control system shown in Fig. 2 . With a number of mathematical manipulations, the output voltages and current commands of the BISSG can be obtained in two-axis stationary reference frame as briefly addressed below.

From Fig. 2, the following voltage and current equations can be easily obtained on KVL.

$$
\begin{aligned}
& L \frac{d I_{O A}}{d t}=V_{A N}-V_{a n}-V_{n N} \\
& L \frac{d I_{O B}}{d t}=V_{B N}-V_{b n}-V_{n N} \\
& L \frac{d I_{O C}}{d t}=V_{C N}-V_{c n}-V_{n N}
\end{aligned}
$$

The above (1)-(3) can be rewritten into (4) and the equivalent forms in two-axis reference frame as expressed in (5) and (6).

$$
\left[\begin{array}{l}
L \frac{d I_{o A}}{d t} \\
L \frac{d I_{o B}}{d t} \\
L \frac{d I_{o C}}{d t}
\end{array}\right]=\frac{2}{3}\left[\begin{array}{ccc}
1 & -\frac{1}{2} & -\frac{1}{2} \\
-\frac{1}{2} & 1 & -\frac{1}{2} \\
-\frac{1}{2} & -\frac{1}{2} & 1
\end{array}\right]\left(\left[\begin{array}{l}
V_{A N} \\
V_{B N} \\
V_{C N}
\end{array}\right]-\left[\begin{array}{c}
V_{a n} \\
V_{b n} \\
V_{c n}
\end{array}\right]\right)
$$

Using Clarke's transformation technique, (4) can be converted into (5) and (6) as follows.

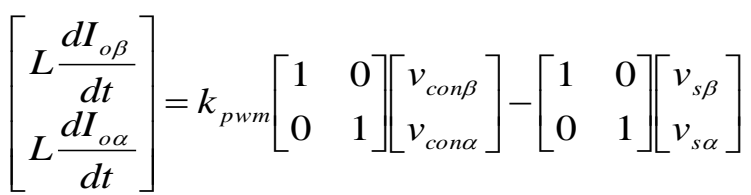

$$
\begin{aligned}
& {\left[\begin{array}{l}
I_{o \beta} \\
I_{o \alpha}
\end{array}\right]=\frac{k_{p w m}}{s L}\left[\begin{array}{ll}
1 & 0 \\
0 & 1
\end{array}\right]\left[\begin{array}{l}
v_{c o n \beta} \\
v_{c o n \alpha}
\end{array}\right]-\frac{1}{s L}\left[\begin{array}{ll}
1 & 0 \\
0 & 1
\end{array}\right]\left[\begin{array}{l}
v_{s \beta} \\
v_{s \alpha}
\end{array}\right]}
\end{aligned}
$$

It is clear that the charging and discharging of the BBESS in the proposed BISSG can be achieved by regulating the real power $(\mathrm{P})$ coming in and out of the three-phase inverter in BISSG, while the output reactive power (Q) can also be simultaneously controlled using the following equation (7) if desired.

$$
\left[\begin{array}{l}
i_{o d, P}^{*} \\
i_{o q, Q}^{*}
\end{array}\right]=\left[\begin{array}{l}
i_{d}^{*} \\
i_{q}^{*}
\end{array}\right]=\left[\begin{array}{l}
P / v_{d} \\
Q / v_{d}
\end{array}\right]
$$

It follows that the three-phase current command signals for the proposed BISSG can be derived from (7) by using the inverse Park's and Clarke's transformations. In the open literature, many current control methods for three phase inverter systems have been proposed. Among them, a PWM based control scheme using high-frequency switching has been widely used in many applications. This is due to the fact that the design of output filter becomes simple for eliminating the high-frequency current harmonics. Besides, the typical advantages of PWM based current control method are its simplicity in implementation and the high speed of its current control loop. The block diagram of the proposed current controller based on (7) is shown in Fig. 4. As can be seen from Fig. 4, the input commands to the derived controllers include two parts, i.e., the reactive power to be compensated and the corresponding current components (charging or discharging currents) reflecting the real power of the BISSG. In practice, to achieve a better dynamic performance a feed forward compensating path or other nonlinear control algorithms, e.g., neural networks, sliding mode and fuzzy controllers can be used.

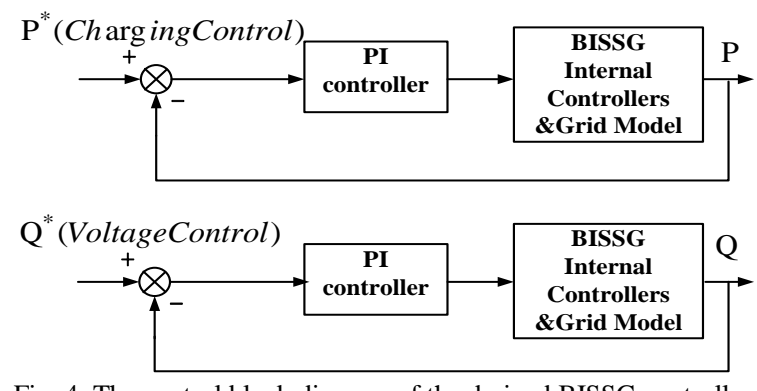

Fig. 4. The control block diagram of the derived BISSG controller.

\section{CAse Studies AND Results}

To investigate the functional feasibility and the dynamic performance of the proposed BISSG control method, a set 
comprehensive simulation studies is carried out on a simple MG distribution network connected with a WTG link and the proposed BISSG as shown in Zone 3 of the MG model (Fig. 1). It is considered that for the whole period of simulations the local loads in Zone 1 and Zone 2 are completely fed by their own local RES based DGs via the micro-grid feeder. In this study case, during the simulation the reactive power designed for the proposed BISSG is assumed not activated. This assumption makes it possible to focus on evaluating the capability of BISSG to track the fast change in the real power current commands required to smooth the output real power of the WTG in Zone 3. It is also assumed that the level of output power from Zone 3 (WTG) is issued by the MGCC via a real-time communication network. To demonstrate a realistic operation scenario in a micro-grid network, three output power conditions are set for the Zone 3 feeder to observe the average charging statuses of the BESS in BISSG. Since the principle of proposed current control technique is based on separating active and reactive current components in synchronous reference frame known as the two-axis components, in all conditions only phase-a parameters (voltage and current) are shown. To demonstrate the performance of the proposed BISSG in smoothing the output power of WTG in Zone 3, the related waveforms regarding feeder real power (reverse power), voltage, current, battery power, current and the DC bus voltage are shown simultaneously. In the following simulation studies, a set of equivalent WTG output power is derived from a recorded wind speed data as shown in Fig. 5. Four typical cases are considered to verify the effectiveness of the proposed BESS based BISSG and its controllers in smoothing the output power of the WTG.

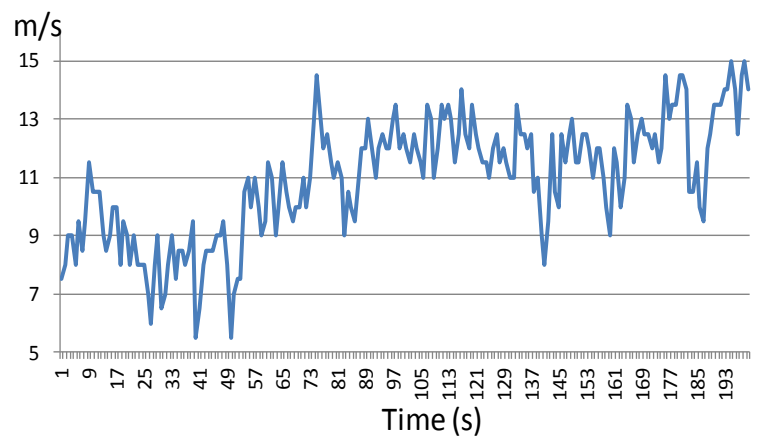

Fig. 5. A set of recorded wind speed data.

Case_1 Description: Connection of WTG and BISSG Link to the Zone 3 of the MG Feeder with Only WTG in Operation.

As mentioned previously, to observe the effects of WTG output power in a practical environment, a set of equivalent WTG output real power derived from a recorded wind speed data as shown in Fig. 5 is injected into the DC interface $(V d)$ of the WTG system as shown in Fig. 3. In the first simulation case, since the BISSG is not in operation the output power regulated by the Inverter 2 is feeding directly towards the feeder of Zone3. Both the simulated and measured results showing the system parameters concerned are shown in Fig. 6 (a) and (b). As can be seen in Fig. 6 (a) (A) and (B), without any smoothing control the output power of the WTG is strongly fluctuating and this may leads to an unacceptable level of voltage and frequency variations in a practical MG.

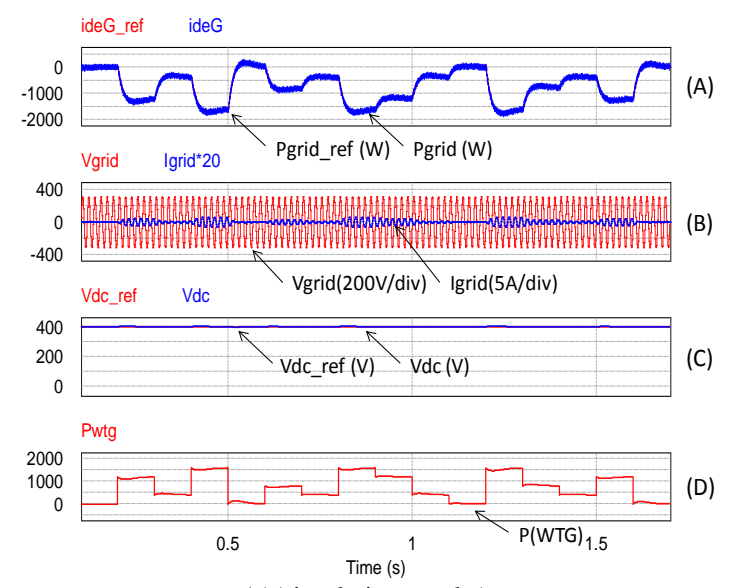

(a)(simulation results)

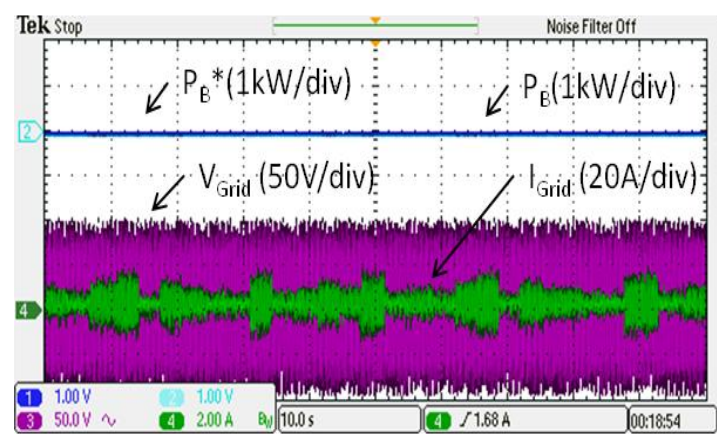

(b) (measured results)

Fig. 6. The related power, voltage, current waveforms of the feeder in Zone3 of the MG system.

Case_2 Description: Connection of WTG and BISSG Link to the Zone 3 of the MG Feeder with Both BISSG and WTG in Operation (Low SOC Case).

In this test case, the BISSG link is connected to the network at $t=0.0 \mathrm{~s}$. At this moment a reverse real power command of $400 \mathrm{~W}$ is also sent to the controller of BISSG at $t=0.0 \mathrm{~s}$. A 16-step changes of wind speed is simulated within 1.6 seconds (the same arrangement as that in simulation case 1). Fig. 7 (a) and (b) show the related powers, voltages and currents waveforms of this study case. As can be seen in Fig. 7 (a) (A) and (B), after the connection of BISSG link to feeder the output current or power of the WTG in Zone3 becomes smooth since the real power fluctuations are fully balanced by the BISSG link with fast charging and discharging control actions shown in Fig. 7 (A) and (C). Since the low SOC of the BESS is assumed in this case the average charging power is higher than the discharging power throughout the simulation period.

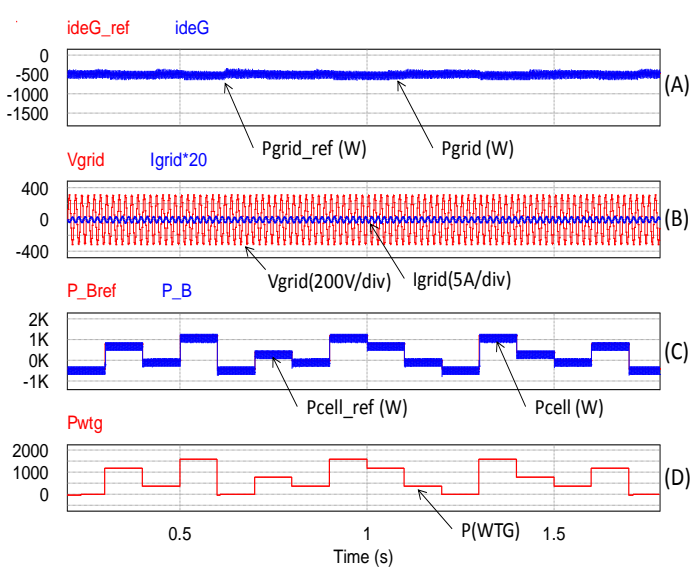

(a)(simulation results) 


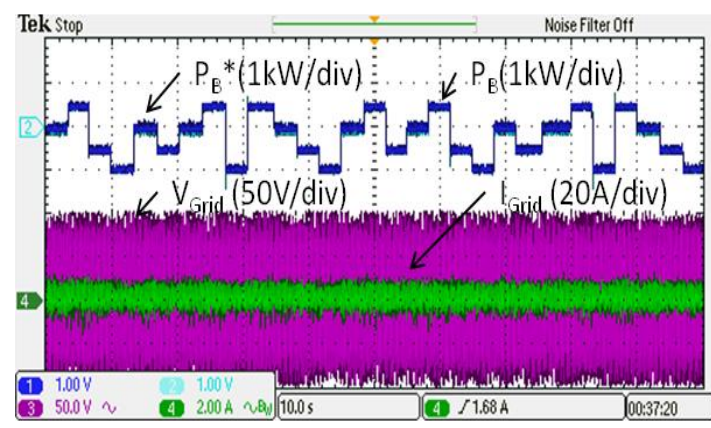

(b) (measured results)

Fig. 7. The related power, voltage, current waveforms of the feeder in Zone3 of the MG system (Pgrid $=400 \mathrm{~W}$ ).

Case_3 Description: Connection of WTG and BISSG Link to the Zone 3 of the MG feeder with Both BISSG and WTG in Operation (Mid SOC Case).

In this test case, the BISSG link is connected to the network at $t=0.0 \mathrm{~s}$. At this moment a reverse real power command of $800 \mathrm{~W}$ is also sent to the controller of BISSG at $t=0.0 \mathrm{~s}$. With the same arrangement, a 16-step change of wind speed is simulated within 1.6 seconds. Fig. 8 (a) and (b) show the related power, voltage and current waveforms of the test case. As can be seen in Fig. 8 (a) (A) and (B), after the connection of BISSG link to feeder the output current or power of the WTG becomes smooth since the real power fluctuations are fully balanced by the BISSG link with fast charging and discharging control actions shown in Fig. 8 (a) (C). Since a mid SOC of the BESS is assumed in this case the average charging and discharging power is evenly regulated.

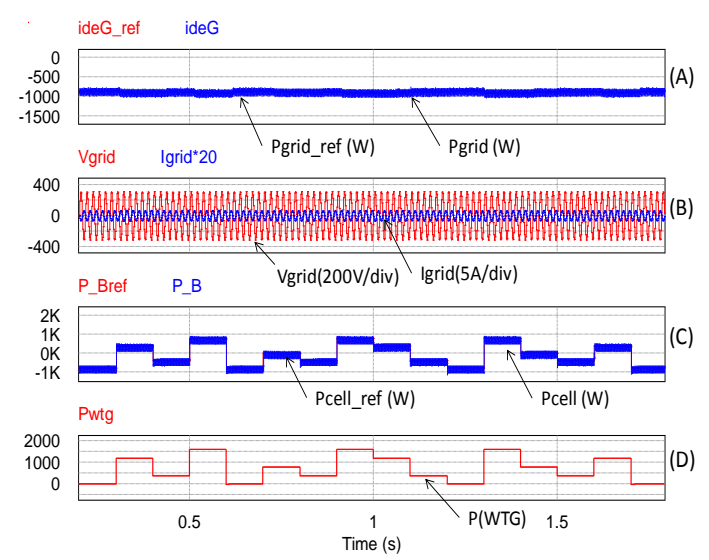

(a) (simulation results)

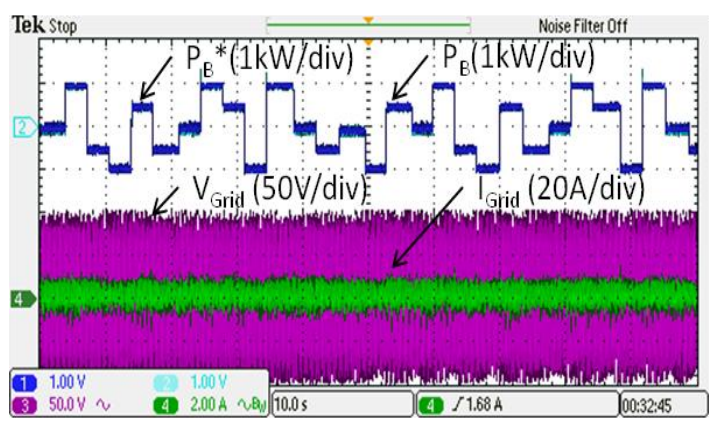

(b) (measured results)

Fig. 8. The related power, voltage, current waveforms of the feeder in Zone 3 of the MG system $($ Pgrid $=800 \mathrm{~W})$.

Case_4 Description: Connection of WTG and BISSG Link to the Zone 3 of the MG Feeder with Both BISSG and WTG in Operation (High SOC Case).

In this test case, the BISSG link is connected to the network at $t=0.0 \mathrm{~s}$. At this moment a real power command of $1200 \mathrm{~W}$ is also sent to the controller of BISSG at $t=0.0 \mathrm{~s}$. A 16 -step changes of wind speed is simulated within 1.6 seconds. Fig. 9 (a) and (b) show the related powers, voltages and currents waveforms of this study case. As can be seen in Fig. 9 (a) (A) and (B), after the connection of BISSG link to feeder the output current or power of the WTG becomes smooth since the real power fluctuations are fully balanced by the BISSG link with fast charging and discharging control actions shown in Fig. 9 (a) (C). Since the high SOC of the BESS is assumed in this case the average charging power is smaller than the discharging power throughout the simulation period.

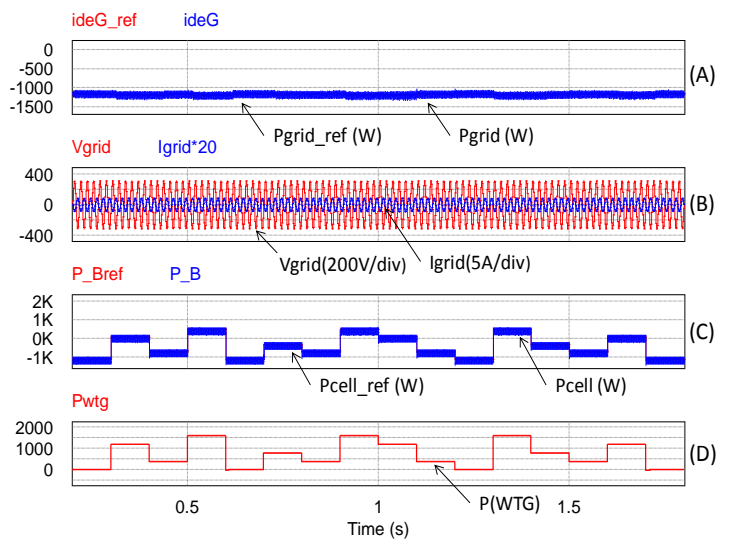

(a) (simulation results)

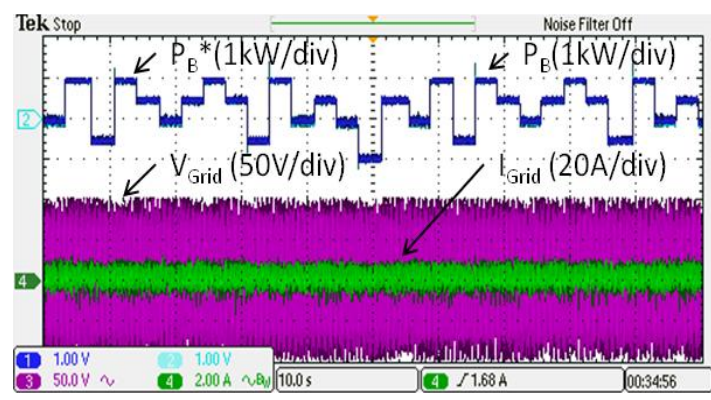

(b) (measured results)

Fig. 9. The related power, voltage, current waveforms of the feeder in Zone3 of the MG system $($ Pgrid $=1200 \mathrm{~W})$.

\section{CONCLUSION}

This paper has demonstrated a feasible battery interfaced static synchronous generator system which can be utilized to smooth the output power flow of grid-tied wind turbine generators. In the proposed control scheme, decoupled current controllers are used in the functional control loops designed for the BISSG, thus better dynamic response has been achieved in various control functions. Based on comprehensive simulation and experimental studies with a simple MG network carried out in PSIM software environment the feasibility and effectiveness of the proposed BISSG control scheme in smoothing the output power of WTG have been proved. It is important to note that in this study, a set of WTG output power derived from the recorded real wind speed data is used in the simulation and experimental studies, which validates the effectiveness of the proposed control strategy in practical applications. In addition, with the fast reactive power control capability the proposed BISSG can also be used to improve the voltage and frequency stability of any RES based MG systems. 


\section{REFERENCES}

[1] X. Wang and H. Liang, "Output characteristics of PV array under different insolation and temperature," in Proc. 2012 Asia-Pacific Power and Energy Engineering Conference, 2012, vol. 1, pp. 1-4.

[2] C. Wang and M. H. Nehrir, "Power management of a stand-alone wind/photovoltaic/fuel cell energy system," IEEE Transactions on Energy Conversion, vol. 23, no. 3, pp. 957-967, 2008.

[3] S. Aghajani, I. A. Joneidi, M. Kalantar, and V. Mortezapour, "Modeling and simulation of a PV/FC/UC hybrid energy system for stand alone applications," IREMOS, vol. 3, no. 1, pp. 82-89, February 2010 .

[4] T. T. Ma and T. H. Shr, "Design of a cost-effective power interface for advanced micro-grid operation and control," in Proc. IMECS 2013 Hong Kong, 2013, pp. 651-656.

[5] Y. Miyamoto and Y. Hayashi, "Evaluation of improved generation efficiency through residential PV voltage control of a clustered residential grid-interconnected PV," in Proc. IEEE PES Innovative Smart Grid Technologies Conference Europe (ISGT Europe), Oct. 2010, vol. 1, pp. 1-8.

[6] H. Laukamp, "The new German electric safety standard for residential PV systems," in Proc. The 25 $5^{\text {th }}$ IEEE Photovoltaic Specialists Conference, 1996, pp. 1405-1408.

[7] J. Carr, J. C. Balda, and A. Mantooth, "A high frequency link multiport converter utility interface for renewable energy resources with integrated energy storage," in Proc. Energy Conversion Congress and Exposition (ECCE), Sept.12-16, 2010, pp. 3541-3548.

[8] IEEE Standard for Interconnecting Distributed Resources with Electric Power Systems, IEEE Standard 1547-2003, July 2003.

[9] R. H. Lasseter, A. Akhil, C. Marnay et al., "The CERTS microgrid concept," White Paper for Transmission Reliability Program, Office of Power Technologies, U.S. Department of Energy, April 2002.

[10] A. Larsson, "Flicker emission of wind turbines during continuous operation," IEEE Transactions on Energy Conversion, vol. 17, no. 1, pp. 114-118, March 2002.

[11] W. Hu, Z. Chen, Y. Wang, and Z. Wang, "Flicker mitigation by active power control of variable speed wind turbines with full scale back-to-back power converter," IEEE Transactions on Energy Conversion, vol. 24, no. 3, pp. 640-649, Sept. 2009.

[12] C. S. Demoulias and P. Dokopoulos, "Electrical transients of wind turbines in a small power grid," IEEE Transactions on Energy Conversion, vol. 11, no. 3, pp. 636-642, Sept. 1996.

[13] C. T. Ma, S. J. Chiang, K. K. Jen, and K. F. Chen, "Investigation on smoothing DG power flow in practical micro-grid operation and control," IJARER, vol. 2, issue 4, pp. 711-719.
[14] H. Nikkhajoei and R. H. Lasseter, "Distributed generation interface to the CERTS microgrid," IEEE Transactions on Power Delivery, vol. 24, no. 3, pp. 1598-1608, 2009.

[15] C. W. Chang and Y. R. Chang, "Energy storage systems for seamless mode transfer in microgrid," in Proc. IEEE Conference PEDS 2011, Singapore, 2011, pp. 799-802.

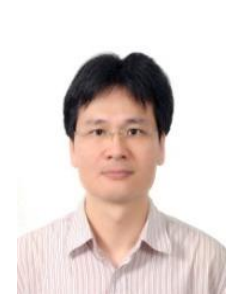

Chao-Tsung Ma received his B.S. degree in electrical engineering from the National Taiwan University of Science and Technology, Taipei, Taiwan R.O.C. in 1989 and his M.S. and Ph.D. degrees from the Department of ECE, the University of Missouri, Columbia, USA in 1992 and the Department of EEE, the University of Strathclyde, Glasgow, UK in 2000, respectively. He has become a member of IEEE since 2003. His employment experiences include the R\&D engineer in Geo-Tech Company, Taiwan and many years in teaching technical courses in the fields of power systems and applications of power electronics. He is currently a member in the editorial board of eight international journals, i.e., IJPSO, IJEEE, IJEE, IJARER, AJEEE, IJTEPE, AIE and frontiers in energy systems and policy. He is also the head of Applied Power Electronics Systems Research Group (APESRG) in the Department of Electrical Engineering, CEECS, National United University, Miao-Li, Taiwan. His special research fields of interest include applied power electronics, custom power, distributed generations, micro-grids, power quality, power system control and flexible ac transmission systems (FACTS).

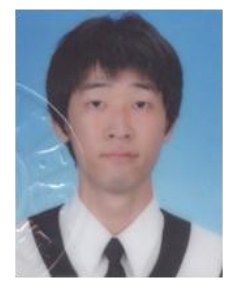

Tzung-Han Shr received his bachelor degree in optical engineering from National United University, Miao-Li, Taiwan, in 2011. He is currently a postgraduate student in the Dept. of Electrical Engineering, National United University, Taiwan and working for his master degree in power engineering field. His research interests include hardware design and implementation of electronic circuits, DSP controlled systems, applied power electronic systems, distributed generations and control of parallel operated systems and grid-tied micro-grids. 